\title{
Towards objective quantification of traditionally subjective clinical assessments: The dichotomy between clinical science and healthcare delivery.
}

\author{
Harsimran S Baweja*
}

Physical Therapy and Applied Movement Sciences, School of Exercise and Nutritional Sciences, College of Health and Human Services, San Diego State University, USA

\section{Introduction}

We live in the era of evidence-based medicine where technology adds or could add a layer of objectivity to quantification, assessment and evaluation of symptoms. This evidence is provided through critical, systematic, scientific inquiry into the disease process and its underlying mechanisms. These discoveries then help clinicians and scientists alike to develop sensitive and reliable measures for diagnosis and treatment, as part of clinical care for patients. The current standard of care in the neurology and neurorehabilitation scenarios such as motor assessments of tremors, balance, postural sway and control remain subjective and depend on the observer's experience, clinical judgment and occasionally trial and error. While, technology is fast paced and has the potential to augment the clinical measures, it needs to be scientifically validated prior to implementation. On that note, how valid and reliable are the current subjective clinical measures? Nevertheless, scientific method is rigorous, discoveries occur over significant time. Thence, translational implementation to augment patient caredelivery continues to remain a challenge for a multitude of reasons beyond scientific inquiry. Researchers and clinicians continue to look for biomarkers that would identify, diagnose and help determine prognosis and recovery. Furthermore, that creates the need to study the biomarkers and their assistance in understanding the long-term effects of neurological insults, and their progression or recovery. All other things being equal, clinical adoption of technology requires the objective tools to be cost and time effective for the technologically savvy clinician to augment clinical care. So, is there added value in the usage of objective measures to augment the current standard of clinical care?

\section{The case for motor symptom assessment in mild traumatic brain injuries}

Sports-related mild traumatic brain injuries, better known as concussions, are one of the most polarizing issues in the field of sports neurology and neurorehabilitation medicine. Sportsrelated concussions are brain injuries caused by biomechanical forces transmitted to the head during participation in sporting activities. The CDC has labeled it as a silent epidemic, with the ensuing brain damage being unpredictable and often resulting in a multitude of neurological symptoms [1]. Given this unpredictable presentation, medical professionals rely on a battery of subjective sideline clinical assessments in order to determine the occurrence of a concussion. This includes measures of gross symptomology (e.g. blurred vision: "How many fingers am I holding up?" and nausea: "Do you feel lightheaded?"), cognitive function (e.g. working memory and attention: "Do you know where are you are and what is today's date?") and motor ability ("close your eyes and stand on one leg without falling"). However, the science of diagnosing and managing concussions in sport beyond the sidelines continues to evolve; yet a lot is still to be learned.

Significant advancements in biomedical technology have helped to develop metabolic biomarkers for testing the extent of the neurologic insult and prognosis. Advanced imaging techniques, such as functional magnetic resonance imaging and diffusion tensor imaging, bring us a step closer to decoding the brain's inner workings. There are suggestions that concussed brain-activation patterns may remain altered despite 'normal' performance on the current sideline clinical-assessment tools. This normalcy is oft erroneously interpreted as clinical recovery and is empirically counterintuitive. Furthermore, measurements such as these cannot be performed on sidelines, are costly and time consuming to obtain, yet they may be the first step along the path to a diagnostic tool. Nevertheless, when it comes to sideline motor symptom assessments such as balance, postural control and gait, the most widely used and popular clinicalassessment tools remain highly subjective in nature with reduced sensitivity to changes and high dependence on observer judgment [2]. A recent statement by the American Medical Society advised the use of balance testing in concussion protocols [3]. This recommendation was based on reports showing evidence of balance declines following concussion [4]. While not every concussion might lead to deficits in balance, those that do have a remarkable effect on balance and postural sway. Nevertheless, the current measures are only sensitive enough to changes if they are larger that over $60 \%$ from baseline [5]. Hazardous interpretations can be made based on these subjective clinical evaluations that are dependent on the observer's assessment and skill in evaluating visible errors, and become debatable on multiple levels [6]. There are multiple studies that report lingering deficits in postural control and gait- 
deviations following concussions for well beyond 3 months, not just 3-7 days contrary to popular belief. Deficits that have been reported are in people considered clinically recovered from concussions. These deficits include, and are not limited to, diminished postural stabilization and altered stability, sway, speed, and response to perturbations during gait.

While their popularity among clinicians is understandable, given the relative ease of usage of these tests there is a more sophisticated approach to concussion balance testing is the objective measurement of body sway control via the use of force plate devices and accelerometers. Currently, force plate technology is the "gold standard" for balance testing, abstracting the center of pressure (COP) from foot contact forces generated during standing on the plate. COP is a proxy for body sway control and increased center of pressure displacement is a known indicator of balance decline in individuals with traumatic brain injuries, including concussion. Unfortunately, force plate balance testing is only used by $\sim 5 \%$ of sports medicine clinicians who perform balance testing as part of their concussion protocols. And, until recently wasn't available on sidelines due to portability and cost effectiveness. More recently, the Wii Balance Board has been used as a low-end force plate to glean out balance measures. This led to researchers demonstrating a close correlation between the COP signals generated by this "toy" and expensive force plates [2]. Even more recently, development of low-cost force plates has shown that these portable devices can be used to obtain diagnostic sensitivity for concussion that is twice that of the current sideline tests such as the balance error and scoring system [7]. A greater push towards using such transitional technologies should be made throughout the field of neurorehabilitation and sports medicine to provide the highest standard of care for potentially concussed and postconcussion athletes.

Despite the current climate surrounding sports-related concussions, the popularity of crude clinical measures of balance over sensitive testing tools like force plates underscores an overall emphasis on cost and feasibility over obtaining the best, most accurate indicators of balance deficits in injured athletes. A critical question to ask at this time is, "Does the screening, diagnostic or prognostic benefit outweigh the cost of care delivery?" Today's standard of care by far supersedes that of a couple decades ago, yet it lacks in objectivity. Clinical adaptation of reliable objective measurements to augment the decision-making is critical to our progress in healthcare delivery. In the era of evidence-based practice, objective measures should be a norm in the clinical care for patients with concussive and other neurological injuries from the sidelines to hospitals and neurorehabilitation clinics. Indeed, the addition of validated measures will eliminate eyeballometrics, trial and error, and guesswork from the decision process. These will help make clinically meaningful interpretations and augment the standard of care delivery process. Overall, providing significant objective evidence will only eliminate conjecture and clinicians can successfully integrate these into decision-making.

\section{References}

1. Taylor CA, Bell JM, Breiding MJ, et al. Traumatic brain injury-related emergency department visits, hospitalizations, and deaths-United States, 2007 and 2013. MMWR Surveill Summ. 2017;66(9):1-16.

2. Chang JO, Levy SS, Seay SW, et al. An alternative to the balance error scoring system: Using a low-cost balance board to improve the validity/reliability of sports-related concussion balance testing. Clin J Sport Med. 2014;24(3):256-62.

3. Harmon KG, Drezner JA, Gammons M, et al. American medical society for sports medicine position statement: Concussion in sport. Br J Sports Med. 2013;47(1):15-26.

4. Peterson CL, Ferrara MS, Mrazik M, et al. Evaluation of neuropsychological domain scores and postural stability following cerebral concussion in sports. Clin J Sport Med. 2003;13(4):230-7.

5. Finnoff JT, Peterson VJ, Hollman JH, et al. Intrarater and interrater reliability of the balance error scoring system (BESS). PMR. 2009;1(1):50-4.

6. Merritt ED, Schmidt JD, Brown CN, et al. Concussion history and time since concussion do not influence static and dynamic balance in collegiate athletes. J Sport Rehabil. 2016;1-16.

7. Goble DJ, Manyak KA, Abdenour TE, et al. An initial evaluation of the B tracks balance plate and sports balance software for concussion diagnosis. Int J Sports Phys Ther. 2016;11(2):149-55.

\section{*Correspondence to:}

Harsimran S Baweja,

Director, Neuromechanics and Neuroplasticity Lab

Assistant Professor, Physical Therapy and Applied

Movement Sciences

School of Exercise and Nutritional Sciences

College of Health and Human Services

San Diego State University

San Diego

USA

Tel: 6195941312

Fax: 6195941470

E-mail: hbaweja@mail.sdsu.edu 